\title{
Body composition and personality traits in so-Yang type males
}

\author{
Jiwon Yoon ${ }^{1}$, Jiho Nam¹, Chae Hun Leem² and Jong Yeol Kim ${ }^{1 *}$
}

\begin{abstract}
Background: The purpose of the present study was to examine the body composition of So-Yang type males according to Sasang constitutional medicine, which is popular in Korea. Different Sasang constitutional types are associated with different muscle distributions, body shapes, and disease susceptibilities. We used the Sasang Personality Questionnaire (SPQ) as a measure of the temperament of each Sasang type.

Methods: In total, 953 subjects aged over 20 years were recruited in Korea. We collected anthropometric parameters and bioimpedence information from the subjects and administered the SPQ. A logistic regression was conducted to calculate propensity scores.

Results: The percentage of skeletal muscle mass in So-Yang (SY) and non-So-Yang (non-SY) males was $45.8 \pm 2.7$ and $44.2 \pm 3.3$, respectively, before matching and $45.8 \pm 2.6$ and $44.9 \pm 3.0$, respectively, after propensity score matching. The extracellular water (ECW)/intracellular water (ICW) and extracellular water (ECW)/total body water (TBW) ratios and SPQ scores were significantly different between the SY and non-SY types.
\end{abstract}

Conclusions: This study suggested that the SY type may be significantly and independently associated with body composition and could be associated with personality.

Keywords: Body composition, Skeletal muscle mass, Sasang constitutional medicine, Personality

\section{Background}

In recent years, several studies have shown a relationship between high body mass index (BMI) and metabolic syndrome [1]. However, not all obese individuals shows evidence of metabolic syndrome $[2,3]$. Thus, there have been several attempts to identify a correlation between body shape and metabolic risk factors. Typically, people who have metabolic syndrome have been characterized as having apple-shaped (abdominal fat) bodies rather than pear-shaped (peripheral fat) bodies [4]. In contrast to peripheral fat mass, visceral fat mass is strongly associated with obesity-related complications such as cardiovascular disease and Type 2 diabetes [5]. Additionally, waist circumference and the waist-to-hip ratio are used to predict metabolic syndrome [6]. Recently, the existence of a metabolically obese, normal weight phenotype associated with normal weight and obese characteristics was proposed $[7,8]$. Even with a low BMI, there is a high

\footnotetext{
* Correspondence: ssmed@kiom.re.kr

${ }^{1}$ KM Fundamental Research Division, Korea Institute of Oriental Medicine,

1672 Yuseong-daero, Yuseong-gu, Daejeon 34054, Republic of Korea

Full list of author information is available at the end of the article
}

risk for cardiovascular disease in people with a high body fat percentage $[9,10]$. The BMI, waist circumference, and body fat percentage are focused solely on body fat mass and do not take into account the body muscle mass, which accounts for $40-50 \%$ of the body composition. Among body muscle mass, skeletal muscle plays a crucial role in glucose metabolism and insulin resistance [11]. Recent studies have shown correlations between low muscle mass and risk factors for cardiovascular disease such as diabetes, hypertension, and dyslipidemia [12]. A decline in muscle mass has been reported as a risk factor for the development of metabolic syndrome [13]. Moreover, relative muscle loss is associated with insulin resistance and the risk of diabetes [14].

In Korea, Sasang constitutional medicine (SCM) was proposed by Lee Je-ma in 1894. According to his theory, humans can be classified into four typologies, Tae-Yang (TY), Tae-Eum (TE), So-Yang (SY), and So-Eum (SE), which are based on characteristics of temperament, body shape and physiology. SCM has been developed and widely used in clinical traditional Korean medicine and 
the proportion of constitutional medical service in the Korean medical service market was 23.5\% [15-17]. During the last ten-year period, scientific research on SCM has been published in medical journals and actively and vibrantly discussed. One notable observation regarding SCM research is that inheritable aspects of SCM have been investigated through genetic polymorphisms [18-20]. In particular, SCM focuses on the relationship between anthropometric factors, temperament, and disease susceptibility. With regard to constitution, each typology exhibits a different, unique susceptibility to particular disease, indicating that medication should be prescribed based on each individual's Sasang type.

Research on certain types of inherited constitutional body types was categorized by Sheldon. Sheldon categorized humans into three different types: components: endomorph (fatness), mesomorph (musculoskeletal robustness), and ectomorph (linearity). Regarding the association with SCM, endomorph, mesomorph and ectomorph are considered to be closely related to the TE, SY, and SE types, respectively [21]. Recently, many studies have explored the relationship between somatotypes and sports ability from diverse angles. According to those results, the mesomorphic component is dominant in certain types of elite players [22-27]. Additionally, there have been attempts to identify relationships between somatotypes and disease susceptibility. For example, the endomorphic component is related to high blood pressure [28, 29], abdominal obesity [30], coronary artery disease [30], and type 2 diabetes mellitus [31].

Like somatotypes, there have been various studies investigating the association of SCM with BMI [32], abdominal obesity [33], and disease susceptibility [34, 35]. Based on the previous reports, the prevalence and relative risk of obesity [33], hypertension [34], diabetes mellitus [35, 36], and metabolic syndrome [37] are higher in the TE type compared with other types. The SE type tends to have weak digestive function and a higher frequency of digestive disease [38-40]. However, few studies have been performed on the SY type and disease susceptibility. Relative muscle mass is inversely associated with insulin resistance and plays a key role in maintaining physical ability $[14,41]$. Based on these results, it can be assumed that there is a relationship between muscular percentage and the SY type's relatively healthy condition. Thus, in this study, we focused on body fat percentage and skeletal muscle percentage assess the characteristics of the SY type individuals.

\section{Methods}

\section{Subjects}

This cross-sectional study was conducted from 2009 to 2015. All of the questionnaire data and clinical data, including Sasang constitutional types (SCTs), were compiled from the Korea Constitutional Multicenter
Bank (KCMB) of the Korea Institute of Oriental Medicine (KIOM). Using this resource, we collected questionnaire data on 953 adults (20 years old or older) who were admitted to the Asan Medical Center. The present study population comprised a population-based sample selected from healthy subjects recruited through advertisements at Asan medical center. To eliminate potential effects of metabolic syndrome on the analysis, we excluded participants with metabolic syndrome. Metabolic syndrome was defined as when three or more of the following conditions were present: high triglyceride levels $(\geq 150 \mathrm{mg} / \mathrm{dl})$, low high-density lipoprotein cholesterol $(<40 \mathrm{mg} / \mathrm{dl}$ in men, $<50 \mathrm{mg} / \mathrm{dl}$ in women), high blood pressure $(\geq 130 /$ $85 \mathrm{mmHg})$, high fasting blood glucose $(\geq 100 \mathrm{mg} / \mathrm{ml})$, and abdominal obesity (waist circumference $(\geq 90 \mathrm{~cm}$ in men, $\geq 80 \mathrm{~cm}$ in women). Individuals who could not be categorized to identify their SCT, were excluded. After applying these exclusions, a total of 567 individuals were analyzed. This study was approved by the Institutional Review Board of the Asan Medical Center. All participants agreed to join this study, and written informed consent for participation was obtained from every subject.

\section{Data collection}

Blood pressure were measured by standard methods. The blood pressure was measured at rest in the left upper arm. Blood Samples were obtained from the left brachial vein after more than $12 \mathrm{~h}$ of fasting. Triglyceride (TG), highdensity lipoprotein (HDL) cholesterol, and fasting blood glucose levels were measured in a central laboratory.

\section{Sasang constitutional diagnosis}

An integrated diagnostic model developed in a previous study, namely the Sasang Constitutional Analysis Tool (SCAT), was used to classify the subjects into different SCTs based on the probability values for each type [42]. Four individual quantitative data sets, such as facial images, body shapes, voice analysis, and questionnaire responses on personality and physiological symptoms were integrated into a single value SCAT. Once individual levels of all required items were entered into the SCAT system, it presented the percentage of each person's potential to be categorized as a SCT using a multinomial logistic regression analysis. Then, a person's SCT with the highest percentage is determined as the person's final type. The diagnostic accuracy of SCAT was higher than that of QSCC II, which has been commonly used for the classification of SCTs and is widely used in previous researches $[36,43]$. Briefly, the facial images of subjects that were obtained with a digital camera were appropriately processed to extract variables for facial points and contours. Variables for facial points and contours include the following: width, height, areas, angle and depth, and ratio of face shape, forehead, eye, upper eyelid, and nose. 
For the body shape analysis, the following 11 variables were collected: forehead circumference, neck circumference, axillary circumference, chest circumference, rib circumference, waist circumference, pelvic circumference, hip circumference, height, weight and body mass index (BMI). Body measurement data were collected from each subject while wearing light clothing. A subject maintains stable breathing and measurement is made between inspiration and expiration. Height and body weight were measured to the nearest $0.1 \mathrm{~cm}$ and $0.1 \mathrm{~kg}$, respectively. BMI was computed as weight divided by height squared $\left(\mathrm{kg} / \mathrm{m}^{2}\right)$. 8-circumference indices were measured by well-trained observers using standard operating procedures developed for the Korea Constitution Multicenter Study. The circumferences of eight regional sites of the body are measured at the levels of the glabella and opisthion (forehead circumference), the thyroid cartilage and cricoid cartilage (neck circumference), the left and right axilla (axillary circumference), the left and right nipple points (chest circumference), the left and right 7th and 8th prominence of costochondral junction (rib circumference), the umbilical cord (waist circumference), the left and right anterior superior iliac spines (pelvic circumference), and the upper edge of the pubis (hip circumference). Voice analysis was performed using the Hidden Markov Model Toolkit (HTK) and Praat voice-analysis programs. A voice signal with a minimum duration of $40 \mathrm{~ms}$ was selected for feature extraction. More than 200 features from the vowels and the sentences were extracted as an initial set, and 88 features were finally selected for a final diagnostic model after applying a genetic algorithm-based feature selection technique The questionnaire for SCTs consisted of 67 multiple-choice questions that included personality characteristics, general temperament, eating habits (e.g., whether having regular meals, frequency of eating meals a day and eating speed), and physiological symptoms (e.g., perspiration, excrement, discomfort in the body, location of discomfort during illness, and existence of fatigue). Sasang constitution questionnaire was developed by KIOM standardized and validated in 2009 and demonstrated good reliability $[44,45]$. Cronbach's alpha was 0.801 in the personality index and Cronbach's alpha was 0.598 in the case of the physiological symptoms index [46]. Thus, the internal consistency of the questionnaire was confirmed.

\section{Bioimpedence assessments}

Skeletal muscle mass, body fat mass, and weight were measured by multi-frequency bioelectric impedance analysis (Inbody 770, eight-point tactile electrode methods, Biospace Co. Ltd., Seoul, Korea). This system uses an electrical current at different frequencies (5, 50, 250, 500, and $1000 \mathrm{kHz}$ ) to directly measure the amount of extracellular and intracellular water in the body. Four electrodes were placed on the palm and thumb of both hands, and four electrodes were placed on the anterior and posterior aspects of the soles of both feet. Based on these impedance values, skeletal muscle mass (SMM) (kg), body fat mass (BFM) $(\mathrm{kg})$, intracellular water (ICW) $(\mathrm{kg})$, extracellular water $(\mathrm{ECW})(\mathrm{kg})$ and total body water (TBW) (kg) were calculated. Body composition parameters based on these variables were defined as follows, to estimate not only the effect of absolute mass but also the effect of the relative ratio on body composition.

SMM (kg) was converted to SMM percentage (skeletal muscle mass/body weight $\times 100$ )

BFM (kg) was converted to BFM percentage (body fat mass/body weight $\times 100$ )

\section{Sasang personality questionnaire}

The Sasang Personality Questionnaire (SPQ) is a 14-item self-report questionnaire developed for the measurement of psychological traits of the Sasang constitutions. The SPQ has the following three personality subscales: SPQ-Behavior (SPQ-B), SPQ-Emotion (SPQ-E), and SPQ-Cognition (SPQ-C). The SPQ-B measures the behavioral component (passive vs. active); the SPQ-E measures the emotional level (static vs. dynamic); and the SPQ-C measures cognition and decision making (meticulous vs. easy going). The SPQ score is the total score of the components of these three subscales. Its structural validity and clinical reliability have been reported $[47,48]$, and the internal consistency values of the SPQ, SPQ-B, SPQ-C, and SPQ-E were 0.81, 0.74, 0.62 , and 0.62 [47], respectively. The total SPQ score and the scores of each subscale were found to increase in order from the SE to TE to SY Sasang types $[47,48]$.

\section{Statistical analysis}

All statistical analyses were performed using SPSS version 23.0 (SPSS, Chicago, IL, USA). All $p$-values $<0.05$ were considered statistically significant. TY type subjects were unavoidably excluded due to the extremely small sample size of the TY type. Since the population of TY is extremely low, SY can represent Yang group and TE and SE can be categorized into same Yin group. Subjects in the SY and non-SY groups were matched 1:1 using propensity scores. The physical characteristics were matched using a propensity score consisting of age, height, weight, and BMI. We calculated the propensity score for each SY and non-SY type using multivariate logistic regression in males and females separately. A matching process was conducted with a minimum distance scoring method, and each propensity score of a SY type was matched with the closest propensity score of a non-SY type. Specifically, matching was limited to a caliper width of 0.05 . Figure 1 shows the change in propensity score distribution between the matched SY and 

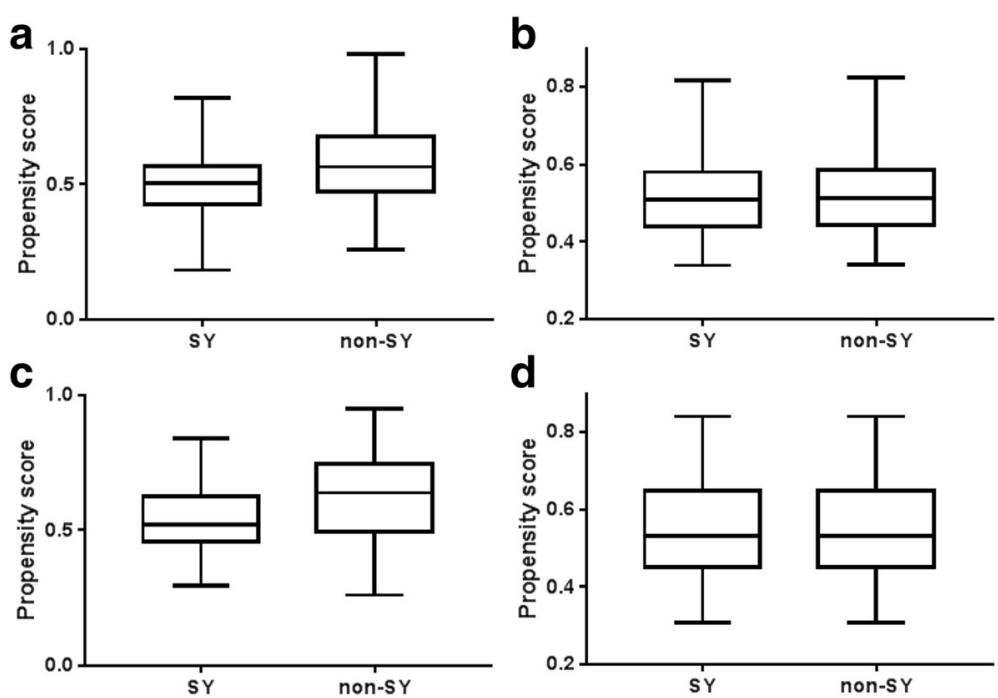

Fig. 1 Comparision of the propensity scores between the SY and non-SY types before and after propensity matching: a Male propensity score before matching, b Male propensity score after matching, c Female propensity score before matching, and $\mathbf{d}$ Female propensity score after matching. SY: So-Yang; non-SY: Tae-Eum and So-Eum

non-SY groups. Data are expressed as the means \pm standard deviations. Between-group comparisons were performed using the independent-samples $t$-test.

\section{Results}

\section{General characteristics of the study population}

Table 1 shows the general characteristics of the participants enrolled in this study. The number of subjects in the original SY and non-SY groups was 126 and 150 in males and 119 and 172 in females, respectively. The general characteristics such as weight and BMI were significantly different between the SY and non-SY types before matching $(p<0.001)$. After propensity score matching, the number of SY and non-SY subjects in each group was 94 males and 97 females, with no statistically significant differences in the general characteristics between the groups.

\section{Body composition index}

Gender-specific values for the body composition index are shown in Table 2. Because non-SY types had significantly increased weight and BMI as shown in Table 1, general body composition values (BFM, SMM, ICW, ECW, and TBW) were higher in the non-SY types compared with the SY type. However, when converted to body weight, both males and females with the SY type had a significantly $(p<0.001)$ greater SMM percentage. These differences remained significant $(p=0.021)$ after propensity matching for age, height, weight and BMI in males. The BFM percentage was lower in the SY group in both males and females, but after propensity matching, this difference became insignificant. Compared to the non-SY types, both males and females with the SY type had significantly lower
ECW/ICW and ECW/TBW ratios before and after propensity matching.

\section{SPQ scores}

The SPQ-B, SPQ-E, and SPQ-C scores were found to be significantly different between types. A t-test was conducted to examine differences between the SY and non-SY types. Before matching, the SPQ-B, SPQ-E, and SPQ-C scores of the SY type $(12.01 \pm 2.31,7.82 \pm 1.99$, and $10.25 \pm 1.81$, respectively) were significantly higher than those of the non-SY types $(10.10 \pm 2.41,7.12 \pm 2.00$, and $9.69 \pm 2.05$, respectively), in males (Fig. 2a). After propensity score matching, the SPQ-B, SPQ-E, and SPQ-C scores of the SY type $(11.96 \pm 2.31,7.84 \pm 1.90$, and $10.21 \pm 1.82)$ were still significantly higher than those of the non-SY types (9.89 $\pm 2.34,6.94 \pm 1.99$, and $9.42 \pm 1.99$, respectively), in males (Fig. 2b). Before matching, the SPQ-B, SPQ-E, and SPQ-C scores of the SY type $(11.96 \pm 2.05,8.75 \pm 1.86$, and $10.61 \pm 2.04$, respectively) were significantly higher than those of the non-SY types $(10.29 \pm 2.60,7.67 \pm 2.13$, and $9.20 \pm 2.28$, respectively), in females (Fig. 2c). After propensity score matching, the SPQ-B, SPQ-E, and SPQ-C scores of the SY type $(11.98 \pm 2.02,8.70 \pm 1.90$, and $10.65 \pm 2.16$, respectively) were still significantly higher than those of the non-SY types $(10.14 \pm 2.67,7.65 \pm 2.02$, and $9.18 \pm 2.40$, respectively), in females (Fig. 2d).

\section{Discussion}

Previous research demonstrated differences in the physical capacities of different Sasang constitutions. Ko et al. reported that the ability to convert energy into muscle strength and speed is greater in the SY type than in 
Table 1 General characteristics according to the SC before and after propensity score matching

\begin{tabular}{|c|c|c|c|c|c|c|}
\hline \multirow[t]{2}{*}{ Variables } & \multicolumn{3}{|c|}{ Before matching } & \multicolumn{3}{|l|}{ After matching } \\
\hline & SY type & non-SY type & $p$ & SY type & non-SY type & $p$ \\
\hline \multicolumn{7}{|l|}{ Male } \\
\hline$n$ & 126 & 150 & & 94 & 94 & \\
\hline Height (cm) & $172.4 \pm 5.7$ & $173.4 \pm 5.6$ & 0.132 & $172.4 \pm 5.8$ & $172.0 \pm 5.5$ & 0.641 \\
\hline Weight (kg) & $69.3 \pm 6.6$ & $73.5 \pm 11.4$ & $<0.001$ & $69.4 \pm 6.7$ & $69.2 \pm 8.3$ & 0.805 \\
\hline BMI (kg/m2) & $23.3 \pm 1.8$ & $24.4 \pm 3.2$ & $<0.001$ & $23.3 \pm 1.8$ & $23.4 \pm 2.6$ & 0.935 \\
\hline Age (yrs) & $34.6 \pm 11.1$ & $36.5 \pm 11.5$ & 0.344 & $35.9 \pm 11.6$ & $37.6 \pm 11.4$ & 0.310 \\
\hline $\mathrm{SBP}(\mathrm{mmHg})$ & $114.4 \pm 13.6$ & $114.6 \pm 12.7$ & 0.891 & $114.8 \pm 13.9$ & $114.2 \pm 12.6$ & 0.736 \\
\hline $\mathrm{DBP}(\mathrm{mmHg})$ & $68.8 \pm 9.4$ & $69.6 \pm 9.2$ & 0.462 & $69.4 \pm 9.6$ & $69.1 \pm 8.7$ & 0.842 \\
\hline Fasting Glucose (mg/dL) & $95.2 \pm 10.1$ & $97.4 \pm 15.7$ & 0.197 & $95.9 \pm 10.8$ & $95.7 \pm 12.9$ & 0.909 \\
\hline $\mathrm{TG}(\mathrm{mg} / \mathrm{dL})$ & $107.1 \pm 98.6$ & $113.1 \pm 62.4$ & 0.544 & $114.2 \pm 112.5$ & $103.5 \pm 42.8$ & 0.406 \\
\hline $\mathrm{HDL}-\mathrm{C}(\mathrm{mg} / \mathrm{dL})$ & $54.4 \pm 13.1$ & $51.7 \pm 12.0$ & 0.078 & $54.6 \pm 13.0$ & $53.1 \pm 11.3$ & 0.402 \\
\hline WC (cm) & $74.0 \pm 9.6$ & $76.0 \pm 9.6$ & 0.062 & $75.0 \pm 8.4$ & $74.9 \pm 8.3$ & 0.951 \\
\hline \multicolumn{7}{|l|}{ Female } \\
\hline$n$ & 119 & 172 & & 97 & 97 & \\
\hline Height (cm) & $158.8 \pm 5.2$ & $160.5 \pm 5.4$ & 0.010 & $159.0 \pm 5.4$ & $159.3 \pm 5.1$ & 0.717 \\
\hline Weight (kg) & $53.9 \pm 4.1$ & $57.5 \pm 8.8$ & $<0.001$ & $54.3 \pm 4.1$ & $54.3 \pm 7.1$ & 0.960 \\
\hline BMI (kg/m2) & $21.4 \pm 1.6$ & $22.3 \pm 3.3$ & 0.001 & $21.5 \pm 1.7$ & $21.4 \pm 3.0$ & 0.908 \\
\hline Age (yrs) & $36.5 \pm 11.1$ & $40.3 \pm 11.8$ & 0.005 & $37.2 \pm 11.4$ & $37.3 \pm 10.9$ & 0.943 \\
\hline $\mathrm{SBP}(\mathrm{mmHg})$ & $102.7 \pm 11.8$ & $105.9 \pm 12.2$ & 0.024 & $103.5 \pm 12.0$ & $103.3 \pm 12.0$ & 0.915 \\
\hline $\mathrm{DBP}(\mathrm{mmHg})$ & $62.5 \pm 7.6$ & $65.0 \pm 8.2$ & 0.008 & $62.8 \pm 7.6$ & $64.0 \pm 7.6$ & 0.250 \\
\hline Fasting Glucose (mg/dL) & $89.4 \pm 13.2$ & $91.9 \pm 11.5$ & 0.091 & $89.6 \pm 14.5$ & $91.1 \pm 11.6$ & 0.450 \\
\hline $\mathrm{TG}(\mathrm{mg} / \mathrm{dL})$ & $76.2 \pm 32.3$ & $83.1 \pm 35.5$ & 0.096 & $77.7 \pm 33.5$ & $78.2 \pm 30.5$ & 0.923 \\
\hline $\mathrm{HDL}-\mathrm{C}(\mathrm{mg} / \mathrm{dL})$ & $64.8 \pm 14.7$ & $63.2 \pm 12.7$ & 0.346 & $64.7 \pm 14.4$ & $62.7 \pm 11.9$ & 0.298 \\
\hline$W C(\mathrm{~cm})$ & $76.2 \pm 5.8$ & $80.4 \pm 8.7$ & $<0.001$ & $76.4 \pm 5.9$ & $77.9 \pm 8.2$ & 0.154 \\
\hline
\end{tabular}

Data shown are the means \pm SDs or numbers. After matching, the data shown are the means \pm SDs. SC sasang constitution, SY so-yang, non-SY: Tae-Eum and So-Eum; BMI body mass index, SBP systolic blood pressure, DBP diastolic blood pressure, TG triglyceride, HDL-C high-density lipoprotein cholesterol, WC waist circumference

other types [49]. Pham et al. indicated that maximal oxygen consumption $\left(\mathrm{VO}_{2 \max }, \mathrm{mL} \cdot \mathrm{kg}^{-1} \cdot \mathrm{min}^{-1}\right)$, a measure of cardiorespiratory fitness is highest among SY type males [50]. He also suggested that increased BMI in the SY type is related to elevated energy expenditure but not to reduced physical endurance [51]. We assumed that the physical capacity and cardiorespiratory fitness of the SY type is associated with body composition. Additionally, the SY body composition may be associated with biopsychological level. In summary, SY males had a relatively high percentage of skeletal muscle mass and low $\mathrm{ECW} / \mathrm{ICW}$ and ECW/TBW ratios compared with the other types, with this tendency maintained after propensity score matching.

The general characteristics of subjects differed markedly depending on height, weight, BMI and age. Because body composition is affected by age and weight, we concluded that these differences could generate bias in the groups. Therefore, the subjects were matched in the SY and non-
SY groups using the propensity score matching method (94 male subjects, 97 female subjects). After matching, there was no difference in height, weight, BMI, and age. This meant that selection bias were minimized between SY and non-SY groups and all groups became almost homogeneous.

Many studies have been performed on body composition of Sasang constitutions using the bioelectric impedance analysis method. However, the majority of them focused on body fat percentage, which has consistently been reported to be greater in the TE type [36, 49-52]. The TE type tends to have a higher BMI; therefore, the lean body mass of the TE type has been reported to be greater than that of other types [36, 49-51]. These findings are consistent with the expectation that a heavier person requires greater muscle mass for movement and has more muscle than a lean person [53]. Thus, we converted absolute skeletal muscle mass $(\mathrm{kg})$ to percentage skeletal muscle mass (skeletal muscle mass/body weight 
Table 2 Body composition index according to the SC before and after propensity matching

\begin{tabular}{|c|c|c|c|c|c|c|}
\hline \multirow[t]{2}{*}{ Variables } & \multicolumn{3}{|c|}{ Before matching } & \multicolumn{3}{|l|}{ After matching } \\
\hline & SY type & non-SY type & $\mathrm{p}$ & SY type & non-SY type & $p$ \\
\hline \multicolumn{7}{|l|}{ Male } \\
\hline BFM (kg) & $13.2 \pm 3.6$ & $16.2 \pm 6.4$ & $<0.001$ & $13.1 \pm 3.6$ & $14.1 \pm 4.7$ & 0.115 \\
\hline SMM (kg) & $31.7 \pm 3.2$ & $32.3 \pm 4.2$ & 0.165 & $31.8 \pm 3.1$ & $31.0 \pm 3.5$ & 0.093 \\
\hline BFM percentage (\%) & $18.9 \pm 4.5$ & $21.5 \pm 5.9$ & $<0.001$ & $18.7 \pm 4.3$ & $20.0 \pm 5.2$ & 0.057 \\
\hline SMM percentage (\%) & $45.8 \pm 2.7$ & $44.2 \pm 3.3$ & $<0.001$ & $45.8 \pm 2.6$ & $44.9 \pm 3.0$ & 0.021 \\
\hline $\mathrm{ICW}$ & $25.8 \pm 2.4$ & $26.3 \pm 3.2$ & 0.161 & $25.9 \pm 2.3$ & $25.3 \pm 2.7$ & 0.098 \\
\hline ECW & $15.4 \pm 1.4$ & $15.8 \pm 1.8$ & 0.055 & $15.5 \pm 1.4$ & $15.2 \pm 1.5$ & 0.200 \\
\hline TBW & $41.2 \pm 3.8$ & $42.1 \pm 5.0$ & 0.109 & $41.4 \pm 3.7$ & $40.5 \pm 4.2$ & 0.126 \\
\hline ECW/ICW & $0.597 \pm 0.015$ & $0.601 \pm 0.014$ & 0.028 & $0.598 \pm 0.015$ & $0.602 \pm 0.014$ & 0.048 \\
\hline ECW/TBW & $0.374 \pm 0.006$ & $0.375 \pm 0.006$ & 0.028 & $0.374 \pm 0.006$ & $0.376 \pm 0.005$ & 0.047 \\
\hline \multicolumn{7}{|l|}{ Female } \\
\hline BFM (kg) & $15.0 \pm 3.1$ & $17.5 \pm 5.9$ & $<0.001$ & $15.1 \pm 3.1$ & $15.8 \pm 5.0$ & 0.264 \\
\hline SMM (kg) & $21.1 \pm 2.0$ & $21.6 \pm 2.6$ & 0.062 & $21.2 \pm 2.0$ & $20.8 \pm 2.3$ & 0.159 \\
\hline BFM percentage (\%) & $27.1 \pm 5.6$ & $29.8 \pm 6.3$ & $<0.001$ & $27.0 \pm 5.9$ & $28.6 \pm 5.9$ & 0.077 \\
\hline SMM percentage (\%) & $38.8 \pm 4.6$ & $37.5 \pm 5.3$ & 0.021 & $38.7 \pm 4.9$ & $38.1 \pm 5.1$ & 0.363 \\
\hline ICW & $17.7 \pm 1.5$ & $18.1 \pm 2.0$ & 0.068 & $17.8 \pm 1.6$ & $17.5 \pm 1.7$ & 0.152 \\
\hline $\mathrm{ECW}$ & $10.9 \pm 1.0$ & $11.2 \pm 1.2$ & 0.006 & $10.9 \pm 1.0$ & $10.9 \pm 1.1$ & 0.546 \\
\hline TBW & $28.6 \pm 2.5$ & $29.3 \pm 3.2$ & 0.028 & $28.8 \pm 2.5$ & $28.3 \pm 2.8$ & 0.260 \\
\hline ECW/ICW & $0.615 \pm 0.013$ & $0.622 \pm 0.013$ & $<0.001$ & $0.615 \pm 0.013$ & $0.622 \pm 0.013$ & $<0.001$ \\
\hline ECW/TBW & $0.381 \pm 0.005$ & $0.383 \pm 0.005$ & $<0.001$ & $0.381 \pm 0.005$ & $0.383 \pm 0.005$ & $<0.001$ \\
\hline
\end{tabular}

Data shown are the means \pm SDs. After matching, the data shown are the means \pm SDs. SC sasang constitution, SY so-yang, non-SY Tae-Eum and So-Eum, BFM body fat mass, SMM: skeletal muscle mass ICW intracellular water, ECW extracellular water, $T B W$, total body water
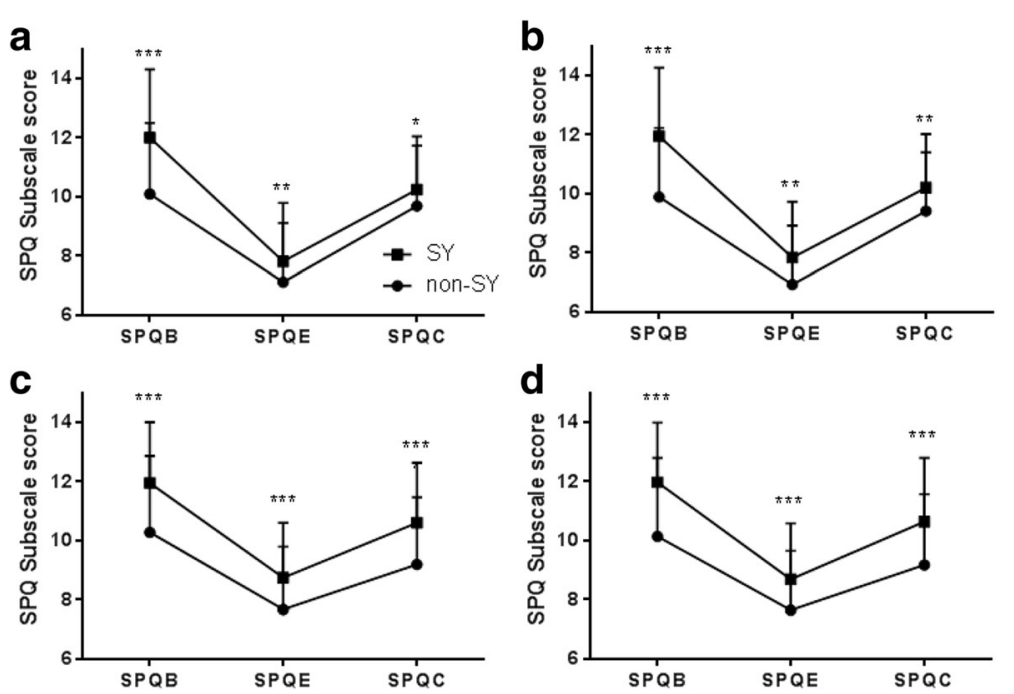

Fig. 2 The SPQ subscale scores of the SY and non-SY types. a The SPQ subscale scores of the male subjects before propensity score matching, b the SPQ subscale scores of male subjects after propensity score matching, $\mathbf{c}$ the SPQ subscale scores of female subjects before propensity score matching, and $\mathbf{d}$ the SPQ subscale scores of female subjects after propensity score matching. SPQ: Sasang Personality Questionnaire; SPQB: SPQ behavior; SPQE: SPQ emotion; SPQC: SPQ cognition; SY: So-Yang, non-SY: Tae-Eum and So-Eum 
$\times$ 100). The SMM percentage was high in the SY male group only. Skeletal muscle plays an important role in physical strength, stamina, and balance [54]. Moreover, a low percentage of skeletal muscle mass is related to insulin resistance, diabetes, and metabolic syndrome [13]. In accordance with these results, the present study demonstrates that SY males have excellent body composition and skeletal muscle distribution. This reinforces the hypothesis that physical capacity and aerobic fitness of the SY type is associated with body composition.

The ECW/ICW and ECW/TBW ratios of the SY type were lower than those of the non-SY types. Compared with obese groups, relatively low ECW/ICW and ECW/ TBW hydration ratios were reported in lean groups [55]. Low ECW/ICW and ECW/TBW ratios for the SY type reflects a significantly higher percentage of fat free mass of the SY type. These results are consistent with the finding that the ECW/ICW ratio of adipocytes is greater than that of fat free mass cells [56]. In this study, low $\mathrm{ECW} / \mathrm{ICW}$ and ECW/TBW ratios of the SY type were maintained even after controlling BMI and age. Therefore, it is a strong indication that SY type shows a unique characteristic of body fluid distribution.

Studies have generally shown personality, especially extraversion, to be positively associated with physical activity [57]. Moreover, physical activity increases the development and maintenance of lean muscle mass [58]. Personality is correlated with muscle strength and is mediated by physical activity level [59]. Because the theological basis of SCM depends on insight into the variation in human psychological differences, it can be suggested that personality traits play an important role in an individual's muscle mass percentage. Therefore, we analyzed the SPQ according to type to assess the presence of such tendencies. The SPQ is a 14-item self-report survey tool used to measure physiological characteristics from the perspective of the SCM. The SPQ consists of three subscales that measure behavioral (SPQ-B), emotional (SPQ-E), and cognitive (SPQ-C) characteristics of personality. The SY type showed a personality profile of active (behavior), dynamic (emotionality), and easy-going (cognition) personality components, with a high overall SPQ score. In contrast, the non-SY types scored relatively lower on the SPQ, with passive (behavior), static (emotionality), and meticulous (cognition) personality components. This finding was consistent with previous studies [47, 60, 61] that SY type repeatedly showed higher score than non-SY type.

Chae et al. noted that the SY type tends to be more extraverted than other constitutions and linked the variation in personality traits to the dopamine system [52]. Based on the Braverman nature assessment, the SY type was reported to have a close relationship to the dopamine system [62]. Dopamine functioning is known to play an important role in voluntary exercise, spontaneous physical activity [63], and extraversion through reward-seeking behavior $[64,65]$. This finding provides evidence that the association between the extraverted, active, and distinctive body composition profiles of the SY type is due to dopamine function. Thus, these results open up the possibility that biopsychological feature of different SCTs type may influence physical characteristics of SCTs.

This study has several limitations. First, the classification of SCTs principally depends on the variation of psychological diversities. However, we only used SPQ scale as a measurement of psychological differences and it should be replicated again using other objective scale in order to be a comprehensive, multifaceted approach.. Second, we did not control for exercise habits or physical activity level. Third, we did not measure muscle strength, which is known to be a better predictive factor for physical performance than muscle mass [66]. Further investigations aimed at assessing the relationship between the high percentage of muscle mass of the SY type and other physical and physiological characteristics are needed.

\section{Conclusions}

We found that the percentage of skeletal muscle mass was high in SY males. Moreover, these traits can explain the low ECW/ICW and ECW/TBW ratios of SY males. The high SPQ score of SY males can explain the difference between the SY type and other constitutions.

\section{Abbreviations \\ BFM: Body fat mass; BMI: Body mass index; ECW: Extracellular water; ICW: Intracellular water; non- SY: Non-So-Yang; SCM: Sasang constitutional medicine; SMM: Skeletal muscle mass; SPQ: Sasang Personality Questionnaire; SY: So-Yang; TBW: Total body water}

\section{Acknowledgments}

This research was supported by the Bio \& Medical Technology Development Program of the National Research Foundation (NRF) funded by the Ministry of Science, ICT and Future Planning (No. 2006-2005173, 2012-0009829, and NRF-2015M3A9B6027139).

\section{Funding}

Not available.

\section{Availability of data and materials}

All data and materials related to this study are available from the corresponding author

\section{Authors' contributions \\ JY carried out the qualitative data analysis and drafted the manuscript. JN participated in the qualitative data analysis. CL participated in the data collection, and contributed to the interpretation of data. JK conceived this study, was the Principal Investigator, participated in its design and coordination, and contributed to the interpretation of data and content of this manuscript. All authors critically contributed to the final manuscript and approved the final version.}

Ethics approval and consent to participate

The Institutional Review Board of the Asan Medical Center approved the study. All participants received a detailed explanation of the study and provided written informed consent. 


\section{Consent for publication}

Not applicable.

\section{Competing interests}

The authors declare that they have no competing interests.

\section{Publisher's Note}

Springer Nature remains neutral with regard to jurisdictional claims in published maps and institutional affiliations.

\section{Author details}

${ }^{1} \mathrm{KM}$ Fundamental Research Division, Korea Institute of Oriental Medicine, 1672 Yuseong-daero, Yuseong-gu, Daejeon 34054, Republic of Korea. ${ }^{2}$ Department of Physiology, University of Ulsan College of Medicine, 88 43-Gil Olympic-Ro, Songpa-gu, Seoul 05505, Republic of Korea.

\section{Received: 8 September 2016 Accepted: 11 August 2017}

\section{Published online: 22 August 2017}

\section{References}

1. Meigs JB, Wilson PW, Fox CS, Vasan RS, Nathan DM, Sullivan LM, D'Agostino RB. Body mass index, metabolic syndrome, and risk of type 2 diabetes or cardiovascular disease. The Journal of Clinical Endocrinology \& Metabolism. 2006;91(8):2906-12.

2. Koster A, Stenholm S, Alley DE, Kim L, Simonsick EM, Kanaya AM, Visser M, Houston DK, Nicklas BJ, Tylavsky FA. Body fat distribution and inflammation among obese older adults with and without metabolic syndrome. Obesity. 2010;18(12):2354-61.

3. Primeau V, Coderre L, Karelis A, Brochu M, Lavoie M, Messier V, Sladek R, Rabasa-Lhoret R. Characterizing the profile of obese patients who are metabolically healthy. Int J Obes. 2011;35(7):971-81.

4. Lebovitz HE. The relationship of obesity to the metabolic syndrome. International journal of clinical practice Supplement. 2003;134:18-27.

5. Hamdy O, Porramatikul S, Al-Ozairi E. Metabolic obesity: the paradox between visceral and subcutaneous fat. Curr Diabetes Rev. 2006;2(4):367-73.

6. Wang F, Wu S, Song Y, Tang X, Marshall R, Liang M, Wu Y, Qin X, Chen D, Hu Y. Waist circumference, body mass index and waist to hip ratio for prediction of the metabolic syndrome in Chinese. Nutr Metab Cardiovasc Dis. 2009;19(8):542-7.

7. Conus F, Rabasa-Lhoret R, Peronnet F. Characteristics of metabolically obese normal-weight (MONW) subjects. Appl Physiol Nutr Metab. 2007;32(1):4-12.

8. De Lorenzo A, Del Gobbo V, Premrov MG, Bigioni M, Galvano F, Di Renzo L. Normal-weight obese syndrome: early inflammation? Am J Clin Nutr. 2007;85(1):40-5

9. Deurenberg-Yap M, Chew S, Deurenberg P. Elevated body fat percentage and cardiovascular risks at low body mass index levels among Singaporean Chinese. Malays and Indians Obesity Reviews. 2002:3(3):209-15.

10. Deurenberg-Yap M, Schmidt G, Van Staveren W, Deurenberg P. The paradox of low body mass index and high body fat percentage among Chinese, Malays and Indians in Singapore. Int J Obes Relat Metab Disord. 2000;24(8): 1011-7.

11. DeFronzo RA, Tripathy D. Skeletal muscle insulin resistance is the primary defect in type 2 diabetes. Diabetes Care. 2009;32(suppl 2):S157-63.

12. Kim Y, Han B-D, Han K, Shin KE, Lee H, Kim TR, Cho KH, Kim DH, Kim YH, Kim H. Optimal cutoffs for low skeletal muscle mass related to cardiovascular risk in adults: the Korea National Health and nutrition examination survey 2009-2010. Endocrine. 2015;50(2):424-33.

13. Moon S-S. Low skeletal muscle mass is associated with insulin resistance, diabetes, and metabolic syndrome in the Korean population: the Korea National Health and nutrition examination survey (KNHANES) 2009-2010. Endocr J. 2014;61(1):61-70.

14. Srikanthan P, Karlamangla AS. Relative muscle mass is inversely associated with insulin resistance and prediabetes. Findings from the third National Health and nutrition examination survey. The Journal of Clinical Endocrinology \& Metabolism. 2011:96(9):2898-903.

15. Kim H-J, Lee S-W, Kim J-Y. A study on the present status of constitutional medical care service. Journal of Sasang Constitutional Medicine. 2006;18(3):166-74

16. Kim S, Lee J, Lee S. Study on the present status of constitutional medica care market. Korean J Oriental Physiology \& Pathology. 2009;23(6):1503-7.
17. Jang E-S, Baek Y-H, Lee S-W. The survey on the state and scale of constitutional medical service market in Korea. Journal of Sasang Constitutional Medicine. 2013;25(1):43-50.

18. JY UM, JC JOO, KY KIM, NH AN, KM LEE, HM KIM. Angiotensin converting enzyme gene polymorphism and traditional Sasang classification in Koreans with cerebral infarction. Hereditas. 2003;138(3):166-71.

19. Song KH, Yu S-G, Cha S, Kim JY. Association of the apolipoprotein A5 gene- $1131 \mathrm{~T}>\mathrm{C}$ polymorphism with serum lipids in Korean subjects: impact of Sasang constitution. Evid Based Complement Alternat Med. 2012;2011:598394.

20. Um J-Y, Lee J-H, Joo J-C, Kim K-Y, Lee E-H, Shin T, Hong S-H, Kim H-M Association between tumor necrosis factor-a gene polymorphism and Sasang constitution in cerebral infarction. The American journal of Chinese medicine. 2005;33(04):547-57.

21. Kim JY, Noble D. Recent progress and prospects in Sasang constitutional medicine: a traditional type of physiome-based treatment. Prog Biophys Mol Biol. 2014;116(1):76-80.

22. Gil S, Gil J, Ruiz F, Irazusta A, Irazusta J. Anthropometrical characteristics and somatotype of young soccer players and their comparison with the general population. Biol Sport. 2010;27(1):17-24.

23. Poliszczuk T, Jankowska E, Mankowska M, Poliszczuk D, Omiecinska I. Profile of an ITF Taekwon-do female champion team in terms of Somatotype and body composition. ARCHIVES OF BUDO. 2015;11:173-85.

24. Gutnik B, Zuoza A, Zuozienè I, Alekrinskis A, Nash D, Scherbina S. Body physique and dominant somatotype in elite and low-profile athletes with different specializations. Medicina. 2015;51(4):247-52.

25. Barlow MJ, Findlay M, Gresty K, Cooke C. Anthropometric variables and their relationship to performance and ability in male surfers. European journal of sport science. 2014;14(sup1):S171-7

26. Barbieri D, Zaccagni L, Cogo A, Gualdi-Russo E. Body composition and somatotype of experienced mountain climbers. High altitude medicine \& biology. 2012;13(1):46-50.

27. Huygens W, Claessens A, Thomis M, Loos R. Body composition estimations by BIA versus anthropometric equations in body builders and other power athletes. J Sports Med Phys Fitness. 2002;42(1):45-55.

28. HARLAN WR, OSBORNE RK, GRAYBIEL A. A longitudinal study of blood pressure. Circulation. 1962;26(4):530-43.

29. Badenhorst L, De Ridder J, Underhay C. Somatotype, blood pressure and physical activity among 10-to 15-year old south African boys: the THUSA BANA study. African Journal for Physical, Health Education, Recreation and Dance. 2003:9(3):184-95.

30. Williams SR, Goodfellow J, Davies B, Bell W, McDowell I, Jones E. Somatotype and angiographically determined atherosclerotic coronary artery disease in men. Am J Hum Biol. 2000;12(1):128-38.

31. Buffa R, Floris G, Putzu PF, Carboni L, Marini E. Somatotype in elderly type 2 diabetes patients. Collegium antropologicum. 2007;31(3):733-7.

32. Pham DD, Do J-H, Ku B, Lee HJ, Kim H, Kim JY. Body mass index and facial cues in Sasang typology for young and elderly persons. Evid Based Complement Alternat Med. 2011:2011:749209.

33. Jang E, Baek Y, Park K, Lee S. Could the Sasang constitution itself be a risk factor of abdominal obesity? BMC Complement Altern Med. 2013:13:72.

34. Lee J, Lee J, Lee E, Yoo J, Kim Y, Koh B. The Sasang constitutional types can act as a risk factor for hypertension. Clin Exp Hypertens. 2011:33(8):525-32.

35. Lee T-G, Koh B, Lee S. Sasang constitution as a risk factor for diabetes mellitus: a cross-sectional study. Evid Based Complement Alternat Med. 2009;6(S1):99-103.

36. Cho NH, Kim JY, Kim SS, Lee SK, Shin C. Predicting type 2 diabetes using Sasang constitutional medicine. Journal of diabetes investigation. 2014;5(5):525-32

37. Choi K, Lee J, Yoo J, Lee E, Koh B, Lee J. Sasang constitutional types can act as a risk factor for insulin resistance. Diabetes Res Clin Pract. 2011:91(3):e57-60.

38. Lee MS, Sohn K, Kim YH, Hwang M-W, Kwon YK, Bae NY, Chae H. Digestive system-related pathophysiological symptoms of Sasang typology: systematic review. Integrative Medicine Research. 2013;2(2):39-48.

39. Lee SK, Yoon DW, Yi H, Lee SW, Kim JY, Kim JK, Hong JH, Shin C. So-eum type as an independent risk factor for irritable bowel syndrome: a population-based study in Korea. The Journal of Alternative and Complementary Medicine. 2014:20(11):846-52

40. Lee $M$, Bae NY, Hwang M, Chae H. Development and validation of the digestive function assessment instrument for traditional Korean medicine: Sasang digestive function inventory. Evid Based Complement Alternat Med. 2013;2013:263752. 
41. Park SW, Goodpaster BH, Lee JS, Kuller LH, Boudreau R, De Rekeneire N, Harris TB, Kritchevsky S, Tylavsky FA, Nevitt M. Excessive loss of skeletal muscle mass in older adults with type 2 diabetes. Diabetes Care. 2009;32(11):1993-7.

42. Do J-H, Jang E, Ku B, Jang J-S, Kim H, Kim JY. Development of an integrated Sasang constitution diagnosis method using face, body shape, voice, and questionnaire information. BMC Complement Altern Med. 2012;12:85.

43. Lee SK, Yoon DW, Yi H, Lee SW, Kim JY, Shin C. Tae-eum type as an independent risk factor for obstructive sleep apnea. Evid Based Complement Alternat Med. 2013;2013:910382.

44. Park H-J, Lee S-W, Dong S-O, Thuy TT, Yoo J-H. Reliability of Sasang constitution questionnaire developed by KIOM for Vietnamese. Journal of Sasang Constitutional Medicine. 2014;26(1):64-74.

45. Yoo J-H, Jang E-S, Kim Y-Y, Park K-H, Lee S-W. A study on the reliability assessment of Sasang constitution questionnaire developed by KIOM for Japanese. Journal of Sasang Constitutional Medicine. 2012;24(2):8-18.

46. Kim M-G, Lee H-J, Jin H-J, Yoo J-H, Kim J-Y. Study on the relationship between personality and ordinary symptoms from the viewpoint of Sasang constitution and cold-hot. Journal of Physiology \& Pathology in Korean Medicine. 2008;22(6):1354-1358.

47. Lee SJ, Park SH, Cloninger CR, Kim YH, Hwang M, Chae H. Biopsychological traits of Sasang typology based on Sasang personality questionnaire and body mass index. BMC Complement Altern Med. 2014;14:315

48. Chae H, Lee S, Park SH, Jang E, Lee SJ. Development and validation of a personality assessment instrument for traditional korean medicine: sasang personality questionnaire. Evid Based Complement Alternat Med. 2012;2012:657013.

49. Ko YS, You SE. Comparisons of physical fitness and body composition among Sasang types with and without body mass index as a covariate. Integrative Medicine Research. 2015;4(1):41-7.

50. Pham DD, Lee JH, Park ES, Baek HS, Kim GY, Lee YB, Ku B, Kim JY, Leem CH. Thermoregulatory responses to graded exercise differ among Sasang types. Evid Based Complement Alternat Med. 2015;2015:879272

51. Pham DD, Lee J, Ku BC, Kim YY, Kim JY. Relation between body mass index and resting metabolic rate, cardiorespiratory fitness and insulin sensitivity in Sasang typology for young male persons: an observational study. European Journal of Integrative Medicine. 2012:4(2):e159-67.

52. Chae H, Lyoo IK, Lee SJ, Cho S, Bae H, Hong M, Shin M. An alternative way to individualized medicine: psychological and physical traits of Sasang typology. The Journal of Alternative \& Complementary Medicine. 2003;9(4):519-28.

53. Janssen I, Heymsfield SB, Wang Z, Ross R. Skeletal muscle mass and distribution in 468 men and women aged 18-88 yr. J Appl Physiol. 2000;89(1):81-8.

54. Janssen I, Heymsfield SB, Ross R. Low relative skeletal muscle mass (sarcopenia) in older persons is associated with functional impairment and physical disability. J Am Geriatr Soc. 2002;50(5):889-96.

55. Chamney PW, Wabel P, Moissl UM, Müller MJ, Bosy-Westphal A, Korth O, Fuller NJ. A whole-body model to distinguish excess fluid from the hydration of major body tissues. Am J Clin Nutr. 2007:85(1):80-9.

56. Wang J, Pierson RN Jr. Disparate hydration of adipose and lean tissue require a new model for body water distribution in man. J Nutr. 1976;106(12):1687-93.

57. Rhodes R, Smith N. Personality correlates of physical activity: a review and meta-analysis. Br J Sports Med. 2006;40(12):958-65.

58. Haskell WL, Lee I-M, Pate RR, Powell KE, Blair SN, Franklin BA, Macera CA Heath GW, Thompson PD, Bauman A. Physical activity and public health: updated recommendation for adults from the American College of Sports Medicine and the American Heart Association. Circulation. 2007;116(9):1081.

59. Tolea MI, Terracciano A, Simonsick EM, Metter EJ, Costa PT, Ferrucci L. Associations between personality traits, physical activity level, and muscle strength. J Res Pers. 2012;46(3):264-70.

60. Chae $\mathrm{H}$, Kown Y. Best-fit index for describing physical perspectives in Sasang typology. Integrative Medicine Research. 2015;4(1):20-8.

61. Lee SJ, Park SH, Chae H. Biopsychological structure of yin-Yang using Cloninger's temperament model and carver and White's BIS/BAS scale. PeerJ. 2016:4:e2021.

62. Lee S, Yu J-S, Lee S. A pilot study of psychological traits in the Sasang constitution according to the Braverman nature assessment. Journal of Pharmacopuncture. 2015;18(4):032-7.

63. Garland T, Schutz H, Chappell MA, Keeney BK, Meek TH, Copes LE, Acosta W, Drenowatz C, Maciel RC, Van Dijk G. The biological control of voluntary exercise, spontaneous physical activity and daily energy expenditure in relation to obesity: human and rodent perspectives. J Exp Biol. 2011;214(2):206-29.
64. Depue RA, Collins PF. Neurobiology of the structure of personality: dopamine, facilitation of incentive motivation, and extraversion. Behav Brain Sci. 1999;22(03):491-517.

65. Cohen MX, Young J, Baek J-M, Kessler C, Ranganath C. Individual differences in extraversion and dopamine genetics predict neural reward responses. Cogn Brain Res. 2005;25(3):851-61.

66. Newman AB, Kupelian V, Visser M, Simonsick EM, Goodpaster BH, Kritchevsky SB, Tylavsky FA, Rubin SM, Harris TB. Strength, but not muscle mass, is associated with mortality in the health, aging and body composition study cohort. J Gerontol Ser A Biol Med Sci. 2006;61(1):72-7.

\section{Submit your next manuscript to BioMed Central and we will help you at every step:}

- We accept pre-submission inquiries

- Our selector tool helps you to find the most relevant journal

- We provide round the clock customer support

- Convenient online submission

- Thorough peer review

- Inclusion in PubMed and all major indexing services

- Maximum visibility for your research

Submit your manuscript at www.biomedcentral.com/submit
C) Biomed Central 